Article

\title{
Crystallization of Mefenamic Acid from Dimethylformamide Microemulsions: Obtaining Thermodynamic Control through 3D Nanoconfinement
}

\author{
Catherine E. Nicholson and Sharon J. Cooper* \\ Department of Chemistry, University Science Laboratories, South Road, Durham, DH1 3LE, UK. \\ E-Mail: c.e.nicholson@durham.ac.uk.
}

* Author to whom correspondence should be addressed; E-Mail: sharon.cooper@durham.ac.uk; Tel.: +44-191-3342098; Fax: +44-191-3342051.

Received: 9 August 2011; in revised form: 8 September 2011 / Accepted: 15 September 2011 / Published: 19 September 2011

\begin{abstract}
Recently we showed how crystallization in microemulsions could lead directly to the most stable polymorph, thereby leapfrogging Ostwald's rule of stages. Here we consider in more details the crystallization of mefenamic acid from dimethylformamide microemulsions. Crystallization of mefenamic acid from bulk DMF has previously been shown to produce only the metastable Form II irrespective of the supersaturation or temperature. In contrast, we show that stable Form I can be produced from DMF microemulsions provided the lowest supersaturations that can achieve crystallization are used; these correspond to initial supersaturations that are significantly higher than those commonly used in bulk solution crystallizations, owing to the large decrease in supersaturation that occurs when a nuclei grows in a 3D-nanoconfined droplet. Increasing the supersaturation above the minimum required for crystallization leads to increasing proportions of metastable Form II crystals. In compositions crystallizing a mixture of Form I and Form II crystals, the Form I crystals can nevertheless be obtained exclusively by slowly heating the microemulsions.
\end{abstract}

Keywords: mefenamic acid; crystallization; polymorph; microemulsion; thermodynamic control 


\section{Introduction}

Crystallization within microemulsions differs fundamentally from bulk crystallization because the limited amount of material within a droplet results in the solution supersaturation decreasing substantially as the nucleus grows. This supersaturation decrease leads to a minimum in the free energy, $\Delta F_{\min }^{*}$, for a post-critical nucleus, $r_{\min }^{*}[1,2]$, of nanometer size, see Figure 1 , and if $\Delta F_{\min }^{*}>k T$ the population of the $r_{\min }^{*}$ nuclei, which depends upon the Boltzmann factor $\exp \left(-\Delta F_{\min }^{*} / k T\right)$, will be very low. Crystallization in microemulsions proceeds initially through the material exchange that can occur when two droplets collide with sufficient energy to form a transient dimer. If one of the colliding droplets contains a nucleus, then its surrounding solute concentration will be depleted, and the resulting concentration gradient in the transient dimer produces a flow of solute into this depleted region, allowing the nucleus to grow. Hence, crystallization in microemulsions depends critically upon whether a colliding droplet is likely to contain a nucleus. In particular, if for all droplets $\Delta F_{\min }^{*}>k T$ the population of the $r_{\min }^{*}$ nuclei will be very low and crystallization will be severely hindered, see Figure 1a. Consequently, the microemulsion system can be stabilized due to 3D nanoconfinement; the supersaturation in the microemulsions is sufficiently high that crystallization would occur readily in an unconfined system, but the 3D nanoconfinement leads to such a decrease in supersaturation as a nucleus grows that a nucleus that is stable $\left(\Delta F_{\min }^{*} \leq 0\right)$, or near stable $\left(\Delta F_{\min }^{*}<k T\right)$, cannot form. In contrast, crystallization can proceed readily via the transient droplet dimer mechanism if $\Delta F_{\min }^{*}<k T$ because then a sizeable population of droplets will each contain a (near) stable nucleus. Hence, for crystallization in microemulsions, the governing factor is the population of (near) stable nuclei. This is in stark contrast to crystallization in bulk systems, where the population of critical nuclei determines the crystallization rate.

Figure 1. Example graphs of free energy change, $\Delta F v s$. nucleus size, $r$ for crystallization from a $3 \mathrm{D}$ nanoconfined solution. Polymorph $A$ (red) is stable and polymorph $B$ (blue) is metastable. In (a) crystallization is disfavoured and the supersaturated system is stabilized due to 3D nanoconfinement. In (b) thermodynamic control over crystallization is achieved and only polymorph $A$ will tend to crystallize as only this form has $\Delta F_{\min }^{*}<k T$.

(a)

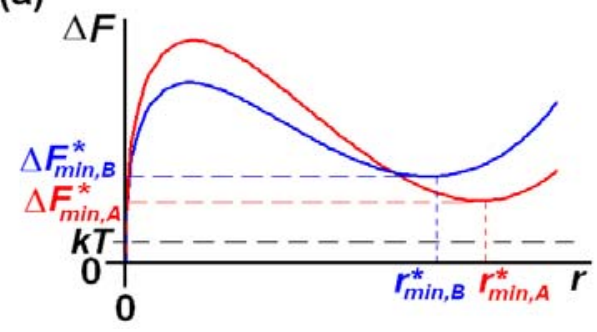

(b)



This presents a unique opportunity for generic thermodynamic control of crystallization in microemulsions [3]. In particular, thermodynamic control is achievable under conditions where crystallization is only just possible in microemulsions, since this corresponds to the case where only the largest droplets with the highest supersaturations can form (near) stable nuclei of (typically) only the stable polymorph [3], see Figure 1b. The stable polymorph would usually be the only form able to 
produce (near) stable nuclei under these conditions because of the following considerations. The stable form has the lowest solubility and so can grow to larger nuclei sizes, $r_{\min }^{*}$, before the supersaturation is depleted. This larger nuclei size, compared to all other polymorphs, and the stable polymorph's intrinsic greater bulk stability, would then typically result in $\Delta F_{\min }^{*}$ having the lowest value. It is, possible, however, that surface effects could result in a metastable form becoming lower in energy, and hence crystallizing out under conditions where crystallization is only just possible. This possibility can be checked by using progressively increasing supersaturations, so that other low energy forms also crystallize, and by crystallizing from microemulsions with different solvents and/or surfactants.

Once (near) stable nuclei have been formed, growth of these nuclei will continue via material exchange during transient droplet dimer formation until the microemulsion's supersaturation is relieved, or the nuclei become larger than the droplets themselves. In the latter case, further crystal growth can then occur either through further collisions with droplets, or via the typically miniscule concentration of their molecules in the continuous phase. Note that although thermodynamic control requires supersaturations for which crystallization is only just possible in the microemulsions, these correspond to initial supersaturations that are typically much higher than would occur in bulk solution crystallizations. This is because the substantial supersaturation decrease in the droplets as the nuclei grow necessitates using very high initial supersaturations to enable (near) stable nuclei to form.

Mefenamic acid (2-[(2,3-(dimethylphenyl)amino] benzoic acid), see Figure 2, has two known polymorphs, Form I and II. Form I is stable at room temperature and has a triclinic unit cell with space

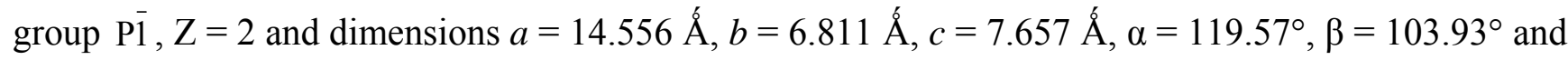
$\gamma=91.30^{\circ}$ [4]. Metastable Form II has a triclinic unit cell with space group $\mathrm{P} \overline{1}, \mathrm{Z}=2$ and dimensions $a=7.6969 \AA, \quad b=9.1234 \AA, c=9.4535 \AA$, $\alpha=107.113^{\circ}, \beta=91.791^{\circ}$ and $\gamma=101.481^{\circ}$ [5]. Crystallization of Form $I$ is readily achieved using solvents such as acetone, dimethylsulfoxide and ethyl acetate [6]. However, crystallization of mefenamic acid from bulk dimethyl formamide (DMF) produces only metastable Form II, irrespective of the rate of crystallization or the crystallization temperature employed [6-8]. This suggests that the nucleation barrier, $\Delta F^{*}$, for stable Form I is significantly higher than that of Form II in DMF.

Figure 2. Mefenamic acid.

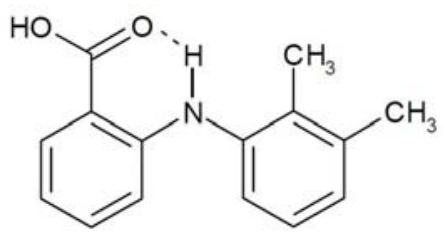

\section{Results and Discussion}

In contrast to the lack of Form I crystallization in bulk DMF, Form I nanocrystals, along with a minority of Form II nanocrystals, were evident from transmission electron microscopy (TEM) analysis of DMF-in-heptane microemulsions containing 3\% by mass of AOT (sodium dioctyl sulfosuccinate) in heptane and 70 to $90 \mathrm{mg} / \mathrm{mL}$ mefenamic acid in DMF on cooling from $50{ }^{\circ} \mathrm{C}$ to $8{ }^{\circ} \mathrm{C}$ over $12 \mathrm{~h}$ and then leaving at $8{ }^{\circ} \mathrm{C}$ for $12-24 \mathrm{~h}$, see Table 1 . These conditions corresponded to the lowest supersaturations at which crystallization was still possible in the microemulsions, with the mean initial 
supersaturation ratios, $\left(\overline{c / c_{\text {sat }}}\right)_{\text {initial }}$, ranging from $\approx 3.0$ to 3.8 . The mean hydrophilic core radii of these microemulsions had previously been determined to be $1.6 \mathrm{~nm}$ [3].

Table 1. Mefenamic acid polymorphs obtained from the DMF microemulsions at $8{ }^{\circ} \mathrm{C}$.

\begin{tabular}{|c|c|c|c|c|c|}
\hline \multicolumn{2}{|c|}{$\begin{array}{l}\text { Mefenamic acid } \\
\text { (MA) in DMF } \\
\text { solution }\end{array}$} & \multirow{2}{*}{$\begin{array}{l}\text { Heptane } \\
\text { /g }\end{array}$} & \multirow{2}{*}{$\begin{array}{l}\mathrm{AOT} \\
/ \mathrm{g}\end{array}$} & \multirow{2}{*}{$\begin{array}{l}\text { \$Mean initial } \\
\text { supersaturatio } \\
\quad \mathrm{n} \text { ratio }\end{array}$} & \multirow[t]{2}{*}{ Polymorph and typical time taken to grow $\sim \mathrm{mm}^{3}$ crystals } \\
\hline $\begin{array}{c}\text { MA in DMF } \\
\mathrm{mg} / \mathrm{mL}\end{array}$ & $/ \mu \mathrm{L}$ & & & & \\
\hline 60 & 20 & 2.91 & 0.09 & 2.6 & Stabilized due to confinement, no $\geq \mu \mathrm{m}$ sized crystals in 6 months \\
\hline $70-75$ & 20 & 2.91 & 0.09 & $3.0-3.2$ & $\begin{array}{c}\text { Mostly ( }>90 \% \dagger) \text { Form I nanocrystals after } 24 \mathrm{~h} \text {, with a minority of } \\
\text { Form II nanocrystals. Growth of the nanocrystals is too low to } \\
\text { observe any macrocrystals even after many months. }\end{array}$ \\
\hline $80-90$ & 20 & 2.91 & 0.09 & $3.4-3.8$ & $\begin{array}{l}\text { Mostly ( }>75 \% \dagger) \text { Form I nanocrystals after } 24 \mathrm{~h} \text {, with a minority of } \\
\text { Form II nanocrystals. Form I macrocrystals typically develop over } \\
\text { weeks to many months. }\end{array}$ \\
\hline $95-100$ & 20 & 2.91 & 0.09 & $4.0-4.2$ & $\begin{array}{l}\text { Form I ( } \sim 40 \text { to } 50 \% \dagger) \text { and Form II nanocrystals after } 24 \mathrm{~h} \text {. Form I } \\
\text { and/or Form II macrocrystals typically develop over weeks to } \\
\text { months. }\end{array}$ \\
\hline $105-120$ & 20 & 2.91 & 0.09 & $4.4-4.9$ & $\begin{array}{c}\text { Mostly ( }(>60 \% \dagger) \text { Form II nanocrystals, with some Form I } \\
\text { nanocrystals after } 24 \text { h. Form II macrocrystals typically develop } \\
\text { over weeks to months. }\end{array}$ \\
\hline
\end{tabular}

\$Mefenamic acid solubility was determined to be $2.3 \%$ by mass in DMF at $8{ }^{\circ} \mathrm{C}$ based on the maximum amount of Form I mefenamic acid powder that could be dissolved in the microemulsion held at $8{ }^{\circ} \mathrm{C}$ in eight weeks. This value is slightly higher than the $1.8 \%$ by mass value quoted in our previous paper [3], and may reflect slight differences in the amount of water non-solvent partitioned into the DMF, combined with slight variations in the exact amount of DMF added to the microemulsion and a longer equilibration time of eight weeks rather than six; $\uparrow$ Based on Fourier transforms of TEM high resolution images.

Form I and Form II are triclinic. However Form I has $\alpha=119.57^{\circ}$, with the $\beta$ and $\gamma$ angles sufficiently close to $90^{\circ}$ and appropriate unit cell length ratios that a near-hexagonal symmetry was

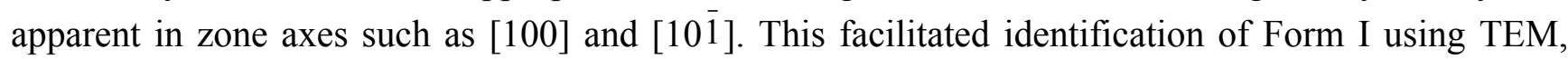
since the crystal structure of Form II could not produce low index zone axes that would give similar near-hexagonal symmetry. The significantly greater unit cell $a$ length for Form I of $14.556 \AA$ also aided its differentiation from Form II. Thus, whilst it was not possible to accurately determine the proportions of Form I and Form II from a powder electron diffraction pattern arising from a cluster of nanocrystals, the diffraction patterns from single nanocrystals and Fourier transforms (FT's) from the HRTEM images of single nanocrystals, could usually be unambiguously attributed to either Form I or Form II. This meant the majority polymorph crystallizing directly from the microemulsions could be reliably identified by TEM. The microemulsions were typically held at $8{ }^{\circ} \mathrm{C}$ for $24 \mathrm{~h}$ before drops from the microemulsions were deposited onto the grids for TEM analysis, with the nanocrystal size observed by TEM ranging from $\sim 10 \mathrm{~nm}$ to $\sim 500 \mathrm{~nm}$. A range of orientations for the Form I and Form II nanocrystals were evident from the TEM analysis. For the Form I nanocrystals, the zone axes found most often were: [100], [101] ], [101], [011] and [111], whilst for Form II the [100] and [101] zones axes occurred most frequently. TEM data obtained from the microemulsions after $24 \mathrm{~h}$ showing $\sim 10-25 \mathrm{~nm}$ nanocrystals and their associated FT's are provided in Figure 3. 
Figure 3. TEM high resolution images (a, c, e and g) and associated Fourier transforms (FT's) (b, d, f, h) of mefenamic acid nanocrystals grown from DMF microemulsions 1 day after cooling to $8{ }^{\circ} \mathrm{C}$. (a) and (c) show images from an $80 \mathrm{mg} / \mathrm{mL}$ mefenamic acid in DMF microemulsion, whilst (e) and (g) show images from a $95 \mathrm{mg} / \mathrm{mL}$ mefenamic acid in DMF microemulsion. The FT's of the red boxed regions in the images enable the nanocrystals to be identified as the following. (a) Form I nanocrystal aligned along the [100] zone axis. (c) Form I nanocrystal aligned along the [011] zone axis. (e) Form I nanocrystal aligned along the [100] zone axis. (g) Form II nanocrystal aligned along the [100] zone axis.

(a)

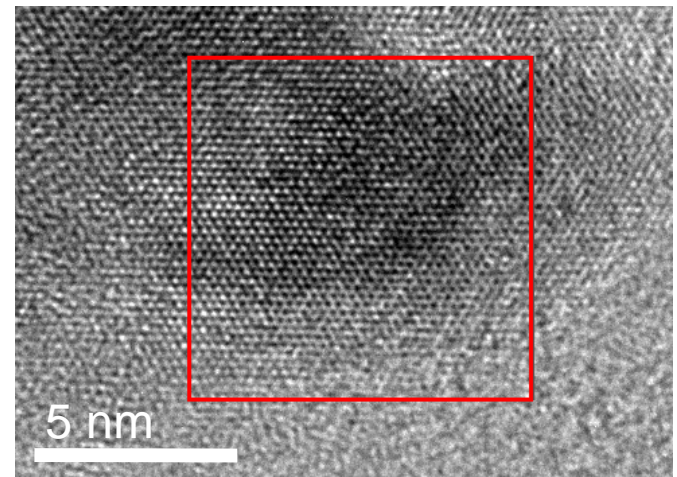

(c)

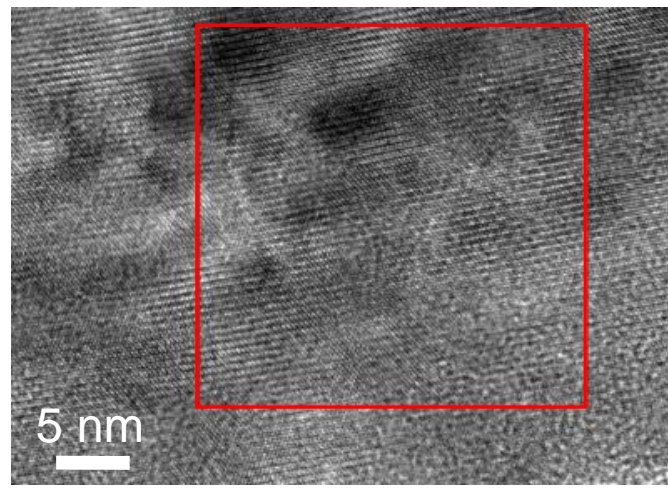

(e)



(g)

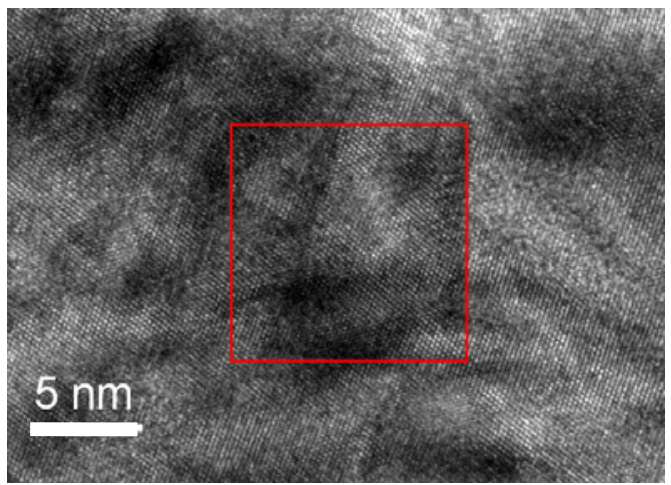

(b)

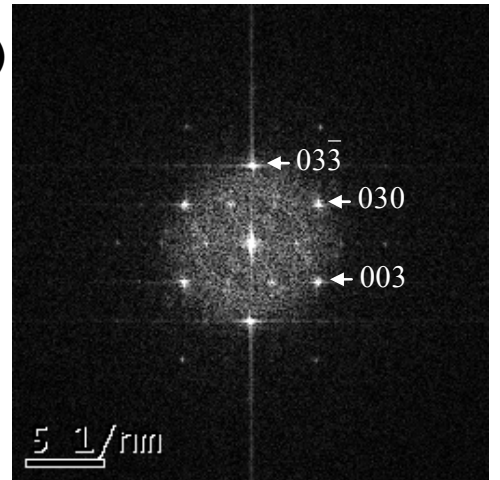

(d)

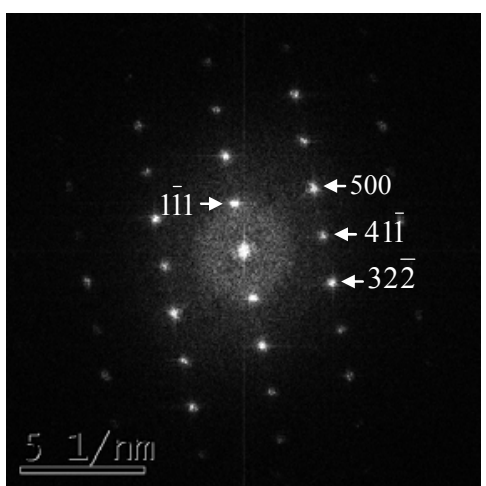

(f)



(h)

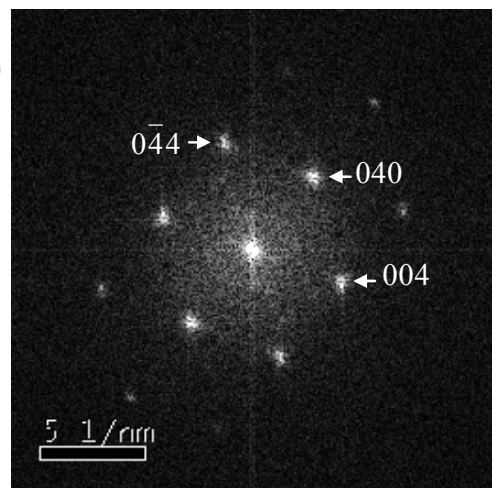


The mean number of mefenamic acid molecules per droplet in the microemulsions was only $\approx 2-3$ for 70 to $120 \mathrm{mg} / \mathrm{mL}$ mefenamic acid in DMF, with $\approx 1.4$ to $2.4 \mathrm{mg}$ mefenamic acid in the whole microemulsion. Hence only a few crystals of length $\sim 0.1$ to $0.5 \mathrm{~mm}$ could ultimately develop from the microemulsions. These larger crystals, visible by the naked eye, grew when the microemulsions were left at $8{ }^{\circ} \mathrm{C}$ for a week or more, though for the lower mefenamic acid in DMF concentration of $80 \mathrm{mg} / \mathrm{mL}$, several months were required for larger crystals to develop. The 70 and $75 \mathrm{mg} / \mathrm{mL}$ microemulsions never developed crystals larger than micron-sized (detectable by optical microscopy) even after several months. The larger crystals were typically of Form I for 80 to $90 \mathrm{mg} / \mathrm{mL}$ mefenamic acid in DMF, with Form II crystals usually obtained from higher concentrations above $100 \mathrm{mg} / \mathrm{mL}$. The Form I crystals grown from these microemulsions had a distinctive needle-like morphology, whereas the Form II crystals were more prismatic, see Figure 4. Figure 5 compares the in-situ Raman spectra of Form I mm-sized crystals grown from microemulsions with $90 \mathrm{mg} / \mathrm{mL}$ mefenamic acid in DMF compared to the Form II spectra obtained from a $120 \mathrm{mg} / \mathrm{mL}$ mefenamic acid in DMF microemulsion. The peaks at 623,702 and $1581 \mathrm{~cm}^{-1}$ are characteristic of Form I spectra, whilst those at 631,694 and $1573 \mathrm{~cm}^{-1}$ signify Form II [6].

Figure 4. TEM images and diffraction patterns of mefenamic acid nanocrystals grown from the microemulsions. (a) Form I nanocrystal grown after 1 day from the $95 \mathrm{mg} / \mathrm{mL}$ mefenamic acid in DMF microemulsion at $8{ }^{\circ} \mathrm{C}$. (b) Diffraction pattern of (a) showing that the crystal is nearly aligned along the [011] zone axis. (c) Form II nanocrystal grown after 1 day from the percolating $70 \%$ AOT $80 \mathrm{mg} / \mathrm{mL}$ mefenamic acid in DMF microemulsion at $8{ }^{\circ} \mathrm{C}$. (d) Diffraction pattern of (c) showing that the crystal is nearly aligned along the [101] zone axis.

(a)

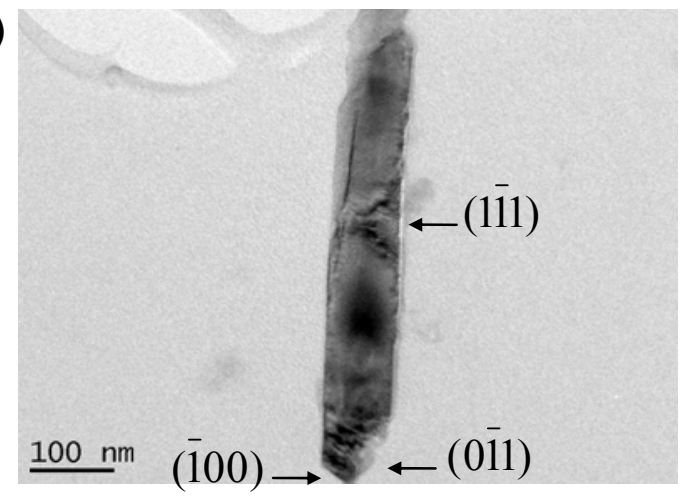

(c)

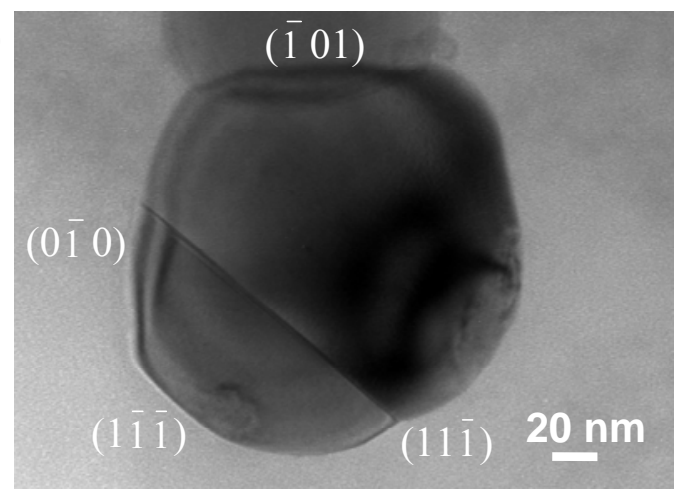

(b)

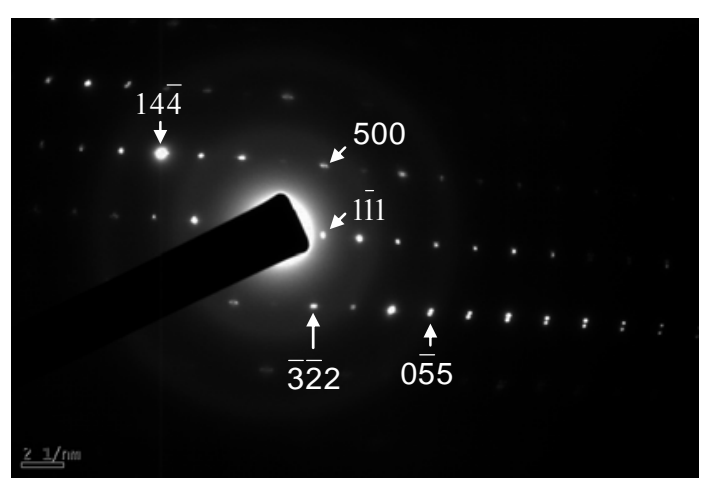

(d)

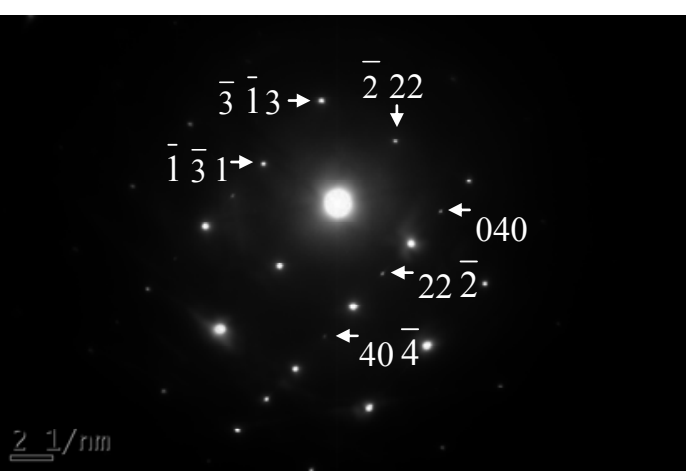


Figure 5. In-situ Raman spectra from (a) 400-1100 $\mathrm{cm}^{-1}$ and (b) $1100-1800 \mathrm{~cm}^{-1}$ showing Form I in red crystallized from a $90 \mathrm{mg} / \mathrm{mL}$ DMF microemulsion compared to Form II in blue crystallized from a $120 \mathrm{mg} / \mathrm{mL}$ DMF microemulsion.

(a)



(b)



TEM analysis on samples with higher mefenamic acid concentrations of 110 to $120 \mathrm{mg} / \mathrm{mL}$ in DMF deposited onto grids after 1-2 days showed that Form I nanocrystals were still present along with the Form II nanocrystals. Consequently it was the faster growth rate of Form II that resulted in only this form subsequently growing to produce $\sim \mathrm{mm}$ sized crystals [9]. The Form II crystals could be preferentially dissolved by gradual heating of the microemulsions to leave just the Form I crystals and/or nanocrystals. Indeed, even when no Form I was evident by visual inspection or optical microscopy, we hypothesise that its presence as sub-micron sized crystals could be inferred by the Form II crystals dissolving in the microemulsions at lower temperatures than the Form II saturation temperature. For instance, at room temperature (typically $<23{ }^{\circ} \mathrm{C}$ ), the solubility of Form II in the microemulsions is $<100 \mathrm{mg} / \mathrm{mL}$ mefenamic acid in DMF, yet Form II crystals grown from 110 and $120 \mathrm{mg} / \mathrm{mL}$ mefenamic acid in DMF at $8{ }^{\circ} \mathrm{C}$ typically completely dissolved on standing the microemulsions at room temperature. Further analysis showed that a temperature of $19{ }^{\circ} \mathrm{C}$ was typically sufficient to dissolve the Form II crystals in these microemulsions. The sub-micron sized 
Form I crystals reduce the mean mefenamic acid solute concentration in the droplets and hence lead to the apparently reduced dissolution temperature of the metastable Form II crystals. Form II is $\sim 1.4$ more soluble in DMF than Form I, this being consistent with the 1.3-1.4 value found in other solvents such as water, ethanol and ethyl acetate [10]. An over-estimate of the size above which Form I crystals would have greater stability than macroscopic Form II crystals can thus be obtained from the GibbsThomson equation using a relative supersaturation value of 1.4 and an interfacial tension value of $\sim 50 \mathrm{mN} \mathrm{m}^{-1}$, i.e., a value that exceeds its likely value. This gives a size $\sim 25 \mathrm{~nm}$, showing that these Form I crystals, which have greater stability than bulk Form II, would indeed be undetectable by visual inspection or optical microscopy.

The TEM analysis conducted on the samples $\sim 24 \mathrm{~h}$ after the mefenamic acid began crystallizing show that the crystallization of stable Form I cannot be attributed to initial crystallization into Form II, followed by its solvent-mediated transformation into the Form I. Indeed, no polymorphic transformation of Form II to Form I is observed in bulk DMF or heptane for unstirred slurries aged for 6 months, highlighting the large nucleation barrier for Form I in DMF. Crystallization of Form I from the microemulsions also cannot be attributed to the presence of AOT and its sodium counter ions, or the water present in the AOT $(\approx 3 \%$ by mass $)$, because viscous percolating microemulsions [11] containing $70 \%$ AOT and the same or higher AOT:mefenamic acid ratios produced a majority of metastable Form II crystals on cooling to $8{ }^{\circ} \mathrm{C}$, even under conditions where crystallization was only just possible, i.e., for percolating microemulsions containing $80 \mathrm{mg} / \mathrm{mL}$ mefenamic acid in DMF. TEM analysis on nanocrystals grown after 1 day in these percolating microemulsions showed that Form I nanocrystals were still present, but the Form II nanocrystals, see Figure 4b, were the majority phase. The faster growth rate of Form II then resulted in only this form growing to $\sim$ mm-sized crystals.. In percolating microemulsions, the dispersed droplets cluster together and can fuse to form transient droplets with much larger droplet sizes [11]. Consequently, the increased difficulty in achieving thermodynamic control in these percolating microemulsions probably arises because a (near) equilibrium concentration of nuclei in the isolated droplets is no longer obtained due to the droplets clustering and fusing to form a proportion of larger droplets in which (near) stable nuclei of metastable Form II can form.

Thermodynamic control of crystallization to produce a majority of Form I crystals occurred in the DMF microemulsions under conditions where crystallization was only just possible, corresponding to 70 to $90 \mathrm{mg} / \mathrm{mL}$ mefenamic acid in DMF at $8{ }^{\circ} \mathrm{C}$. The mean number of mefenamic acid molecules per droplet for these microemulsions was $\approx 2$ and the mean initial supersaturation, $\left(\overline{c / c_{\text {sat }}}\right)_{\text {initial }}$ with respect to Form I was 3.0 to 3.8. However, given crystallization was only just achievable, it would have been initiated in only those droplets with the highest supersaturations, since only these would be able to form the (near) stable nuclei. An estimate of the likely supersaturations in these droplets can be made as follows. Previous work [3] on glycine crystallization from microemulsions suggested that $<10^{-8}$ of the droplets were capable of forming (near) stable nuclei for systems crystallizing at the lowest possible mean supersaturations [12]. If a similar $<10^{-8}$ proportion of droplets formed (near) stable nuclei in the mefenamic acid microemulsions when crystallization was only just possible, then the droplets forming (near) stable nuclei would contain $\geq 14$ mefenamic acid molecules, assuming a Poisson distribution of mefenamic acid molecules amongst the droplets, and so would have initial 
supersaturation ratios $>20$. Even if a higher proportion of $\sim 10^{-5}$ droplets could form (near) stable nuclei, these droplets would contain $\geq 10$ mefenamic molecules and have initial supersaturation ratios $>15$. Consequently it is clear that very large initial supersaturations are required for mefenamic acid crystallization to occur in these DMF microemulsions. This is to be expected given a droplet's supersaturation will decrease substantially as the nucleus within it grows. These large initial supersaturations are crucial for Form I crystallization, since they enable high nucleation barriers to be surmounted. Indeed, it is likely that it is the large nucleation barrier, in particular, that prevents Form I crystallization from bulk DMF. We note, though, that our experiments show Form I grows slower in the DMF microemulsions than Form II, suggesting that Form I would also grow slower in bulk DMF, and this may be a contributing factor to its lack of crystallization in bulk DMF.

From the above analysis, it is likely that the smallest number of mefenamic acid molecules needed to form the (near) stable nuclei under conditions where crystallization is only just possible is $\sim 9-13$, given these nuclei will be surrounded by slightly supersaturated solution containing $\approx 1-2$ mefenamic acid molecules. These values are far lower than the number comprising (near) stable nuclei in unconfined bulk systems under conditions where crystallization is only just possible, where the attainment of critical nuclei, and not (near) stable nuclei, limits the crystallization. The high initial supersaturations that are necessary for thermodynamic control in microemulsions ensure that nucleation barriers to all polymorphic forms are surmountable, so that stable nuclei of the most stable Form I can arise, in contrast to their lack of nucleation in the bulk.

\section{Experimental Section}

All materials were obtained from Aldrich and used without further purification. Ultrapure water with a resistivity of $18 \mathrm{M}^{-1} \mathrm{~cm}$ was provided by a UHQ water filtration unit. The mefenamic acid microemulsions typically contained $3 \mathrm{~g}$ of a $3 \%$ by mass solution of AOT in heptane, together with $20 \mu \mathrm{L}$ of a mefenamic acid-in-DMF solution. Some microemulsions, however, were prepared at a much higher AOT concentration, so that they were percolating. In these microemulsions, $3 \mathrm{~g}$ of a $70 \%$ by mass solution of AOT in heptane were used with $470 \mu \mathrm{L}$ of the mefenamic acid-in-DMF solution to retain the same ratio of surfactant to DMF solution. The mefenamic acid concentration in the DMF

was varied from $70-120 \mathrm{mg} / \mathrm{mL}$, corresponding to $\left(\overline{c / c_{\text {sat }}}\right)_{\text {initial }}=3.0$ to 4.9 in the microemulsions at $8{ }^{\circ} \mathrm{C}$. The microemulsions were prepared by mixing the constituents at elevated temperature $\left(\approx 50-60{ }^{\circ} \mathrm{C}\right.$ for which the mefenamic acid saturation concentration is above $\approx 180 \mathrm{mg} / \mathrm{mL}$ ) and sonicating for typically between 30 seconds to 5 minutes to ensure a clear microemulsion phase was obtained. The mefenamic acid microemulsions were then cooled to $8{ }^{\circ} \mathrm{C}$ over $12 \mathrm{~h}$ and left at $8{ }^{\circ} \mathrm{C}$ to crystallize.

Transmission Electron Microscopy (TEM): Small aliquots of the microemulsions were deposited on a holey carbon grid and examined in a JEOL 2100F field emission gun TEM (FEG TEM) operating at $200 \mathrm{kV}$. Phase contrast high resolution electron microscopy (HREM) was used to image nanometre size crystals. The structure of an individual nanocrystal was determined by indexing the fast Fourier transform, extracted from the appropriate region of the image. 
Raman Microscopy: Raman spectroscopy was the preferred analytical method for determining the mefenamic polymorph of larger crystals because Form I and II are readily distinguishable using this technique, and the crystals could be analysed in situ. In contrast powder X-ray diffraction would require the crystals removal from the microemulsion, drying, and then crushing to produce a powder, and this could potentially cause a polymorph transformation into Form I. Raman spectra were obtained with a LabRAM from Horiba Scientific. The Raman microscope was equipped with a $450 \mathrm{~mW}$ external cavity stabilized diode laser as the excitation source, operating at $785 \mathrm{~nm}$. The unit consisted of an Olympus optical light microscope, motorized translational stage and a CCD camera. The Raman spectra were collected over a range of 400 to $1800 \mathrm{~cm}^{-1}$. Data were processed and analyzed using LabSpec V5.63.

\section{Conclusions}

Whereas crystallization of mefenamic acid from bulk DMF solutions produces crystals of the metastable Form II, we have shown that the stable Form I can be obtained from DMF microemulsions. These results provide an example of the generic thermodynamic control of crystallization that is possible when crystallizing from microemulsions. In particular, thermodynamic control arises under conditions where crystallization is only just possible. Owing to the substantial supersaturation decrease that occurs as a nucleus grows within a microemulsion droplet, the initial supersaturations necessary to achieve this crystallization are significantly higher than those commonly employed in bulk solutions. This is advantageous, as it means high nucleation barriers are surmountable, and so helps ensure crystallization of the most stable polymorph even when this form is not achievable from bulk solution. This generic approach enables the leapfrogging of Ostwald's rule to stages to provide stable polymorphs directly from microemulsions.

\section{Acknowledgments}

We are extremely grateful to Budhika Mendis for performing the electron microscopy. The work is supported by the UK Engineering and Physical Sciences Research Council Grant No. EP/D070228/01.

\section{References and Notes}

1. Reguera, D.; Bowles, R.K.; Djikaev, Y.; Reiss, H. Phase transitions in systems small enough to be clusters. J. Chem. Phys. 2003, 118, 340-353.

2. Shirinyan, A.S.; Wautelet, M. On phase changes in nanosystems. Mater. Sci. Eng. C 2006, 26, 735-738.

3. Nicholson, C.E.; Chen, C.; Mendis, B.; Cooper, S.J. Stable Polymorphs Crystallized Directly under Thermodynamic Control in Three-Dimensional Nanoconfinement: A Generic Methodology. Cryst. Growth Des. 2011, 11, 363-366.

4. McConnell, J.F.; Company, F.Z. N-(2,3-Xylyl)anthranilic acid, $\mathrm{C}_{15} \mathrm{H}_{15} \mathrm{NO}_{2}$, mefenamic acid. Cryst. Struct. Commun. 1976, 5, 861-864.

5. Lee, E.H.; Byrn, S.R.; Carvajal, T.M. Additive-Induced Metastable Single Crystal of Mefenamic Acid. Pharm. Res. 2006, 23, 2375-2380. 
6. Alvarez, A.J.; Singh, A.; Myerson, A.S. Polymorph Screening: Comparing a Semi-Automated Approach with a High Throughput Method. Cryst. Growth Des. 2009, 9, 4181-4188.

7. Aguiar, A.J.; Zelmer, J.E. Dissolution behaviour of polymorphs of chloramphenicol palmitate and mefenamic acid. J. Pharm. Sci. 1969, 58, 983-987.

8. Cesur, S.; Gokbel, S. Crystallization of mefenamic acid and polymorphs. Cryst. Res. Technol. 2008, 43, 720-728.

9. The faster growth rate of Form II in DMF is likely to be due to a specific DMF solvent-polymorph interaction, given that Form I can grow readily in other solvents such as acetone, dimethylsulfoxide and ethyl acetate, and that hydrogen bonded dimers are thought to be the dominant growth form in both polymorphs, see reference [5].

10. Romero, S.; Escalera, B.; Bustamante, P. Solubility behavior of polymorphs I and II of mefenamic acid in solvent mixtures. Int. J. Pharm. 1999 , 178, 193-202.

11. Ray, S.; Moulik, S.P. Dynamics and Thermodynamics of Aerosol OT-Aided Nonaqueous Microemulsions. Langmuir 1994, 10, 2511-2515.

12. This $<10^{-8}$ value arose from estimating that the (near) stable nuclei would need to contain $\sim 20-30$ molecules to provide sufficient thermodynamic preference for the stable $\gamma$-glycine polymorph to crystallize as the majority polymorph, given the $\alpha$-glycine polymorph is only $\sim 0.2 \mathrm{~kJ} \mathrm{~mol}^{-1}$ less stable, and on the assumption that the glycine molecules had a Poisson distribution amongst the droplets.

(C) 2011 by the authors; licensee MDPI, Basel, Switzerland. This article is an open access article distributed under the terms and conditions of the Creative Commons Attribution license (http://creativecommons.org/licenses/by/3.0/). 